\title{
Fabrication of New PCM Hydrogel Composites
}

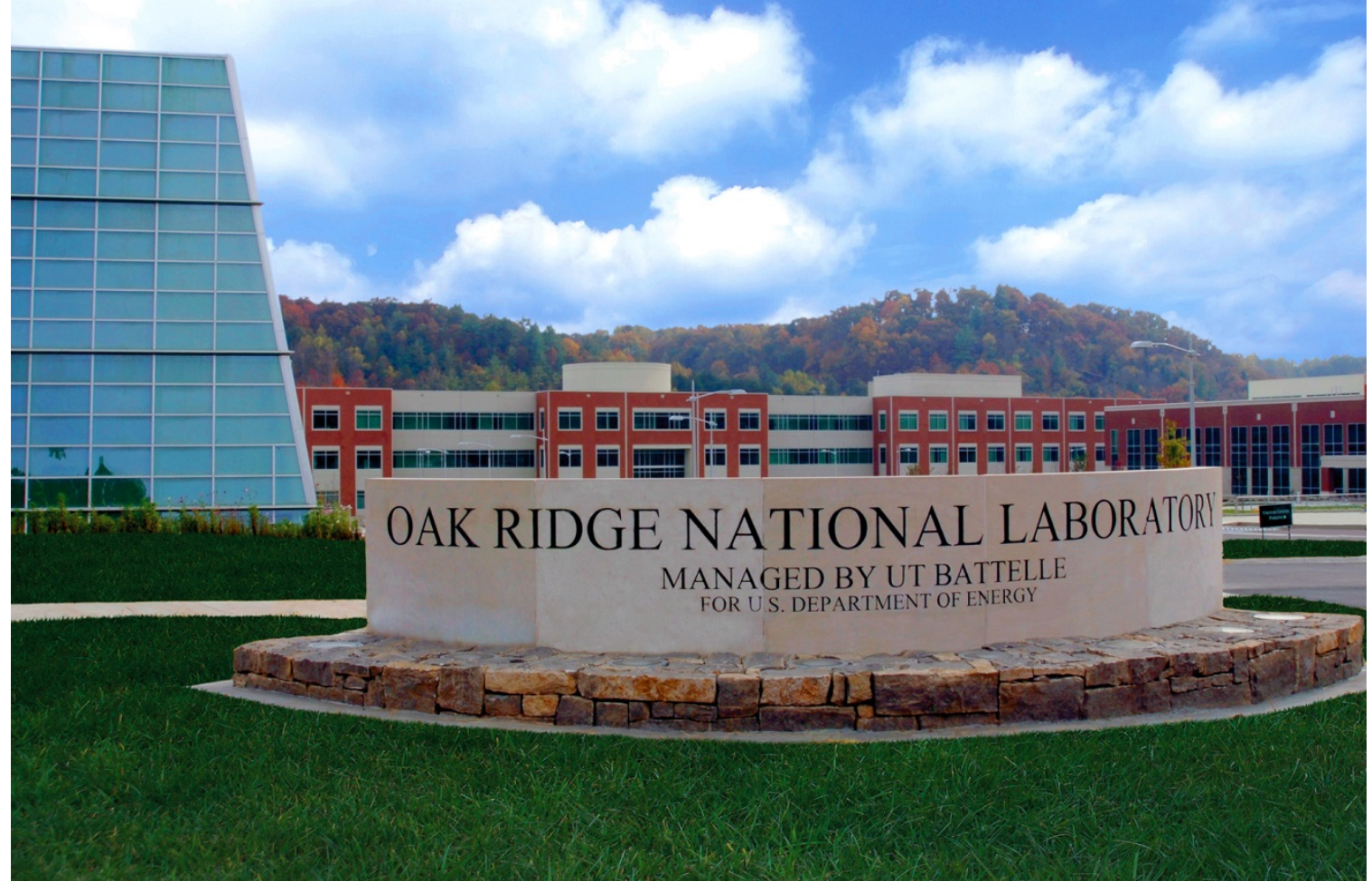

Yuzhan Li

Vera Bocharova

Seung Pyo Jeong

Navin Kumar

Som Shrestha

Kyle Gluesenkamp

Diana Hun

Approved for public release.

Distribution is unlimited.

April 5, 2021 


\title{
DOCUMENT AVAILABILITY
}

Reports produced after January 1, 1996, are generally available free via US Department of Energy (DOE) SciTech Connect.

Website www.osti.gov

Reports produced before January 1, 1996, may be purchased by members of the public from the following source:

\author{
National Technical Information Service \\ 5285 Port Royal Road \\ Springfield, VA 22161 \\ Telephone 703-605-6000 (1-800-553-6847) \\ TDD 703-487-4639 \\ Fax 703-605-6900 \\ E-mail info@ntis.gov \\ Website http://classic.ntis.gov/
}

Reports are available to DOE employees, DOE contractors, Energy Technology Data Exchange representatives, and International Nuclear Information System representatives from the following source:

Office of Scientific and Technical Information

PO Box 62

Oak Ridge, TN 37831

Telephone 865-576-8401

Fax 865-576-5728

E-mail reports@osti.gov

Website https://www.osti.gov/

This report was prepared as an account of work sponsored by an agency of the United States Government. Neither the United States Government nor any agency thereof, nor any of their employees, makes any warranty, express or implied, or assumes any legal liability or responsibility for the accuracy, completeness, or usefulness of any information, apparatus, product, or process disclosed, or represents that its use would not infringe privately owned rights. Reference herein to any specific commercial product, process, or service by trade name, trademark, manufacturer, or otherwise, does not necessarily constitute or imply its endorsement, recommendation, or favoring by the United States Government or any agency thereof. The views and opinions of authors expressed herein do not necessarily state or reflect those of the United States Government or any agency thereof. 


\title{
FABRICATION OF NEW PCM HYDROGEL COMPOSITES
}

\author{
Yuzhan Li \\ Vera Bocharova \\ Seung Pyo Jeong \\ Navin Kumar \\ Som Shrestha \\ Kyle Gluesenkamp \\ Diana Hun
}

April 5, 2021

Prepared by

OAK RIDGE NATIONAL LABORATORY

Oak Ridge, TN 37831-6283

managed by

UT-BATTELLE, LLC

for the

US DEPARTMENT OF ENERGY

under contract DE-AC05-00OR22725 


\section{CONTENTS}

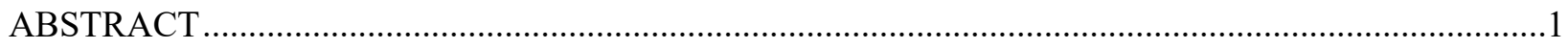

1. PREPARATION CONDITIONS AND EFFECT OF REAGENT CONCENTRATIONS ON

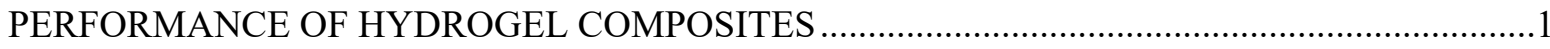

2. CYCLING STABILITY TEST, SUPERCOOLING, AND THERMAL CONDUCTIVITY ...............2 


\begin{abstract}
Salt hydrates are low-cost phase-change materials (PCMs) with excellent thermal energy storage capacities. However, they are limited by low thermal cycling stability due to incongruent melting-induced phase separation. This project will solve the long-standing issue of phase separation of salt hydrates by stabilizing salt particles in a charged polymer hydrogel network. The proposed approach uses combined physical and chemical stabilization mechanisms achieved by selecting a proper chemistry and molecular weight of the polymer matrix. Our approach is different from current stabilization strategies that depend solely on physical confinement.
\end{abstract}

The following elements of the milestone have been fully met:

- Identify hydrogel candidates to be used with sodium sulfate decahydrate and report compatibility test results.

- Report synthesis method and key parameters (e.g., $\mathrm{pH}$, concentration, morphology) controlling the interaction strengths between salt particles and the hydrogel network.

- Report cycling stability test results and document methods for improving thermal conductivity, reducing supercooling $(<6 \mathrm{~K})$, and carrying out scale-up synthesis.

$\circ$ The element of this milestone not yet met is to achieve a thermal conductivity of $>1 \mathrm{Wm}^{-1} \cdot \mathrm{K}^{-1}$.

\title{
1. PREPARATION CONDITIONS AND EFFECT OF REAGENT CONCENTRATIONS ON PERFORMANCE OF HYDROGEL COMPOSITES
}

Among several polymer candidates, we selected an alginate polymer because of its biocompatibility, low cost, ability to effectively trap water, and ability to crosslink with metal ions. The latter characteristic provided the smallest possible pore size, which may benefit the stability of the formulation by preventing leaching of the encapsulate.

We prepared and tested two main systems: (1) a formulation containing alginate polymer and sodium sulfate decahydrate (SSD) and (2) a mixture of alginate with SSD crosslinked with $\mathrm{Fe}^{3+}$ ions. We tested alginates from different commercial sources and found that a sodium alginate from Sigma Aldrich provides the best performance. We characterized the samples using different techniques to identify the molecular weight and its distribution, melting and glass transition temperatures, and viscosity. For the first system, powders of alginate and SSD were mixed in a glass vial and then heated to $50^{\circ} \mathrm{C}$ in an ultrasound bath for about an hour. Then the temperature of the ultrasound bath was set to $20^{\circ} \mathrm{C}$ and the mixture was cooled under sonication. For the second system, alginate and SSD were mixed and heated to $50^{\circ} \mathrm{C}$ in the ultrasound bath for about an hour. After dissolution and homogenization, the solution was added dropwise to an aqueous solution of $\mathrm{FeCl}_{3}(1 \mathrm{mg} / \mathrm{ml})$ that was prepared and placed in the same ultrasound bath. The crosslinked liquid drops immediately precipitated to the bottom of the vial. The excess $\mathrm{FeCl}_{3}$ solution was removed and the resulting material was washed with water and cooled down to room temperature.

For both systems, we demonstrated that the concentrations of components and the preparation methods selected were critical for the interaction interplay in the system that affected the performance and stability of the material. The interaction was indirectly related to the changes in the melting temperature of the composite. The effects of concentrations of iron and alginate on the performance of the system are summarized in Figure 1. In the case of the alginate+SSD mixture (Figure 1, left), the increase in the alginate concentration results in a reduction of the enthalpy of the melting of the composition. 

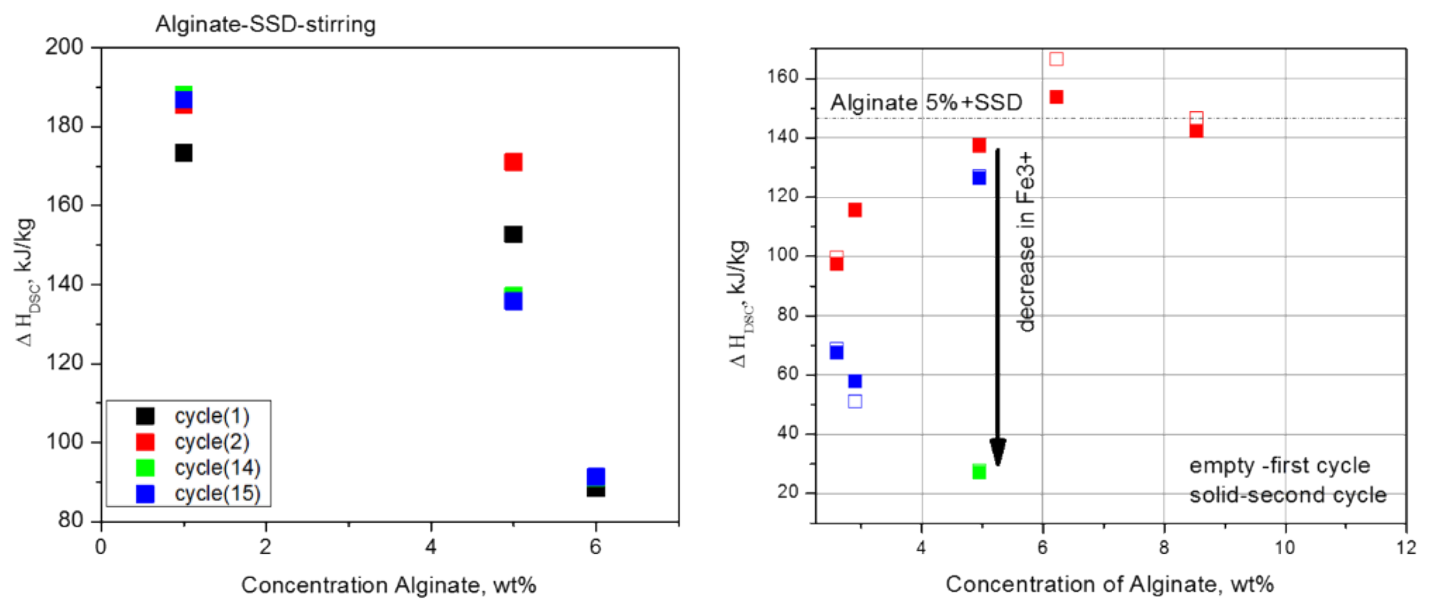

Figure 1. Effects of alginate (left) and alginate /iron (III) (right) concentrations on the enthalpy of melting of SSD. The measurements were performed with differential scanning calorimetry.

The enthalpy of melting was measured by differential scanning calorimetry. In the case of alginate+SSD+ $\mathrm{Fe}^{3+}$ (Figure 1, right), the membrane performance shows a nonlinear dependence on the concentration of alginate. Moreover, we determined that the optimum initial aqueous concentration of $\mathrm{FeCl}_{3}$ was also important; it was found to be $1 \mathrm{mg} / \mathrm{ml}$. Increasing or decreasing the iron (III) solution concentration was shown to deteriorate the membrane performance. It is important to mention that changing the melting temperature with the component concentration exactly mimicked the enthalpy of melting behavior presented in Figure 1. Thus, better performance was found in membranes with higher melting temperatures. The latter result suggests that both alginate and iron interacted with SSD, and the outcome of that interaction was reflected in the changes in the melting temperature. The main conclusion was that experimental conditions that lead to higher melting temperatures will likely result in improved membrane performance.

The preparation methods also had an important influence on the composite performance. The utilization of ultra-strong ultrasound during preparation resulted in a significant reduction in the membrane performance. We hypothesized that this effect, combined with the reduction in the pore size of the SSD, led to stronger exposure of the SSD clusters to interaction with the matrix. In comparison, stirring or weak ultrasound provided reproducible results that partially verified our hypothesis. We will perform structural characterizations of the systems to further verify the hypothesis.

\section{CYCLING STABILITY TEST, SUPERCOOLING, AND THERMAL CONDUCTIVITY}

The cycling stability for all samples presented in Figure 1 was measured using T-history tests. The best results for both formulations are presented in Figure 2. Both of the presented formulations have already partially met the go/no-go milestone, for which a report is scheduled on September 30, 2021. 

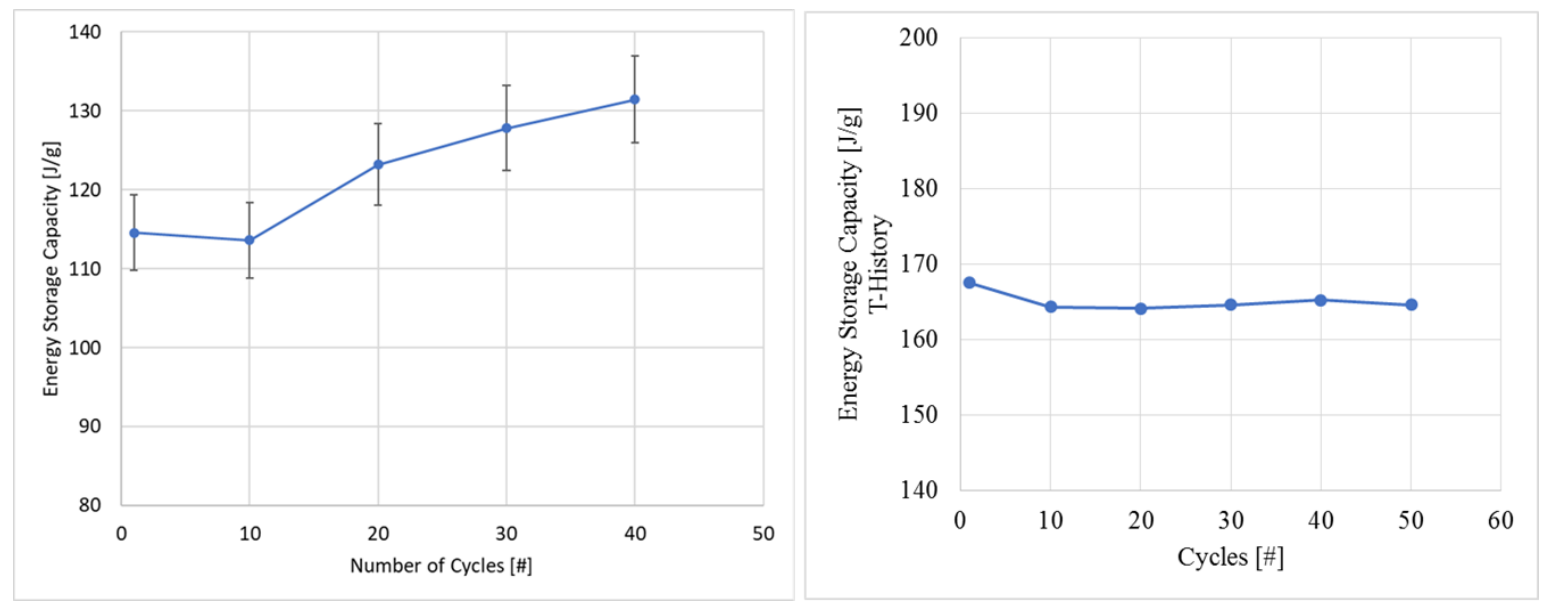

Figure 2. T-history measurements of alginate $5 \%+\mathrm{SSD}+\mathrm{FeCl}_{3}$ (left) and alginate $6 \%+\mathrm{SSD}$ (right). The supercooling is at $3{ }^{\circ} \mathrm{C}$ and the melting at $30^{\circ} \mathrm{C}$ for alginate $5 \%+\mathrm{SSD}+\mathrm{FeCl}_{3}$, and the supercoiling is at $5^{\circ} \mathrm{C}$ and the melting temperature at $32^{\circ} \mathrm{C}$ for alginate $6 \%+\mathrm{SSD}$.

Specifically, the heat capacity performance for a formulation containing $6.5 \mathrm{wt} \%$ of alginate mixed with SSD was tested for 50 cycles and showed highly stable performance and no sign of degradation in the energy storage capacity values. We determined that the incorporation of the polymer and of the iron ions both reduced supercooling. This is an interesting phenomenon that requires further evaluation. The energy storage capacity value for $6.5 \mathrm{wt} \%$ of alginate mixed with SSD was $165 \mathrm{~J} / \mathrm{g}$. In this specific sample, before the measurements, partial SSD salt precipitation was noticed, which did not affect the performance of the sample. That behavior was thought to be associated with the solubility limits of SSD in alginate, which provides room for further improvement in the energy storage capacity. We hypothesize that this issue can be resolved by tunning the polymer concentration. Indeed, comparison of samples with different concentrations of alginate suggested that the performance of the composite tended to improve with increasing alginate concentration. For instance, a change in the concentration from $5 \mathrm{wt} \%$ to $6 \mathrm{wt} \%$ yielded an improvement in energy heat capacity from $150 \mathrm{~J} / \mathrm{g}$ to $165 \mathrm{~J} / \mathrm{g}$. A very conservative estimation suggests that the energy storage capacity can be increased to $180 \mathrm{~J} / \mathrm{g}$ simply by increasing the concentration of alginate to $10 \mathrm{wt} \%$.

Preliminary measurements (based on the transient plane source at Oak Ridge National Laboratory) suggested that the thermal conductivity of $6.5 \mathrm{wt} \%$ of alginate mixed with SSD was $\sim 0.19 \mathrm{~W} / \mathrm{m} \cdot \mathrm{K}^{-1}$. This value is low. We plan to improve the thermal conductivity by adding carbon nanotubes or graphene flakes. The effects of concentration and aspect ratio on thermal conductivity will be evaluated.

Finally, the work showed that sample preparation conditions are fairly simple and can be easily adopted for large-scale production.

In summary, we have identified several important formulations that show good energy storage capacity performance and good cycling stability. The thermal storage capacity can be further improved through further sample optimization. The thermal conductivities of the samples were low, but there are ways to improve it by adding a highly thermally conductive material with a high aspect ratio. The method of preparation is suitable for scale-up. 
